\title{
EL DISCURSO MÉDICO Y LA INVENCIÓN DEL HOMOSEXUAL (ESPAÑA 1840-1915)
}

\author{
Francisco Vázquez García \\ Depto. de Historia, Geografía y Filosofía \\ Universidad de Cádiz
}

\section{RESUMEN}

En este trabajo se examina la recepción de la psicopatología de las perversiones y más específicamente del discurso psiquiátrico sobre la homosexualidad en la España de principios de siglo. Con este objetivo, se analizan los obstáculos y las transformaciones conceptuales, así como las condiciones culturales que hicieron posible la difusión social de estas innovaciones teóricas más allá de los límites de la propia disciplina mental.

PALABRAS CLAVE: homosexualidad, psiquiatría, España, regeneracionismo, perversiones.

\section{SUMMARY}

This paper analyzes the reception of the psychopathology of perversions and, more specifically, the reception of the psychiatric discourse about homosexuality in Spain around 1900. With this aim, we explore obstacles, conceptual transformations and cultural conditions doing posible the social spreading of these theoretical innovations beyond the limits of mental medicine.

KEY WORDS: homosexuality, psichiatrey, Spain, regenerationism, perversions.

\section{INTRODUCCIÓN}

Los anexos 1 y 2 incluidos al final de este trabajo presentan dos relatos, con fecha diferente, que describen en cada caso un diagnóstico - con efectos médico-legalesdonde se implica la relación sexual de varones adultos con niños. Entre ambas narraciones no sólo media un vasto lapso temporal; remiten, respectivamente, a universos conceptuales muy distintos. La diferencia no reside únicamente en el grado de precisión y especialización del lenguaje utilizado, ni en la relación que se establece entre el vocabulario de los legos (los vecinos, el padre, el encargado del local convocados en el primer caso, el «mundo ignorante» mencionado en el segundo) y el del facultativo. Cambia también por completo el ámbito de objetos que se considera relevante para el diagnóstico, la manera misma de modelar la realidad proyectando una determinada representación (esquemas de clasificación, definiciones, conceptos) de la misma. 


\section{FRANCISCO VÁZQUEZ GARCÍA}

En el caso de Montemayor, la locura se relaciona con una explosión de furor, una ofuscación más o menos brusca e intermitente. El desorden sexual cometido queda algo desdibujado en medio de los «gritos y desvergüenzas», el «escándalo», la «fuga», el intento de asesinar al padre. Por otro lado, se estima que la posible locura debería quedar asociada a un defecto de raciocinio («por las contestaciones que se nos ha dado a las preguntas y cuestiones..»); implica la constatación de un delirio. Justamente es la aparente ausencia de éste lo que hace dudar al facultativo a la hora de acreditar la alienación mental del sujeto.

En el caso del sacerdote U.L., el diagnóstico apunta a realidades muy diferentes. La locura interrogada ya no se identifica con una ausencia de razón, sino con perturbaciones del instinto y de la voluntad. La conducta sexual desordenada ocupa ahora el centro de la escena, y se la describe con cierto detalle. Al localizar la enfermedad en la dinámica del instinto se hace posible efectuar una doble inscripción del problema. Por una parte, queda emplazado en la superficie misma del organismo (los «estigmas físicos»); por otra en la cadena de la evolución (los «antecedentes fisiopatológicos de la familia»). La indagación de estos últimos se convierte entonces en primordial. La familia misma, y no tanto el espacio manicomial, invocado en el primer anexo, es el objeto privilegiado de observación. Estas circunstancias implican, en comparación con el caso de Montemayor, un desplazamiento completo del campo conceptual; de los «delirios», las «demencias», las «manías» y las «intermitencias» de la razón se pasa a la «degeneración», la «herencia de los instintos», la «perversión» («manifestaciones psicopáticas homo-sexuales»).

Otro contraste interesante. Tiene que ver con la relación establecida, en cada caso, entre justicia penal y medicina mental. En el episodio de Montemayor se sugiere que la «demencia» convierte al acto en irresponsable. El padre del arrestado sugiere que su hijo es un loco y no un criminal, por eso pide que se le encierre en un asilo para dementes. El diagnóstico de la patología excluye la imputación de responsabilidad. En el relato sobre U.L., cambia por completo el panorama. Se evidencia una controversia entre el veredicto del Jurado y el de la Sala. Ésta, aparentemente más al tanto de la psiquiatría del momento, no veía una incompatibilidad entre la locura — que afectaba en este caso al ámbito de la voluntad y del instinto y no a la razón-y la criminalidad.

¿Qué ha tenido que ocurrir para que se produzca esta transformación en la evaluación psiquiátrica de las relaciones mantenidas por un adulto con menores del mismo sexo?. O dicho de otro modo: ¿qué ha tenido que ocurrir para que la categoría de «perversión homosexual», o simplemente de «homosexualidad» se incorporara en la España de 1900 al saber psiquiátrico y a la cultura sexual del momento? 


\section{Psiquiatría, Medicina Legal E Higiene ANTES de la HomoseXUalidad}

En 1846, cuando las acciones de Montemayor inquietaban a las autoridades, las conductas sexuales eran interrogadas por la medicina española a partir de tres frentes distintos y a la vez íntimamente relacionados entre sí:

En primer lugar el alienismo, la primera forma de medicina mental difundida en España e importada directamente de Francia. En 1847 se tradujo al castellano el Des Maladies Mentales (1838) del insigne Esquirol, verdadera síntesis del pensamiento alienista. Ya unos años antes, esta corriente se había dejado sentir en la comunidad médica española a través de dos hitos principales. En primer lugar la edición, en 1846, de la memoria escrita por José Pérez Villargoitia, De los Remedios para mejorar en España la suerte de los enajenados. En segundo lugar, la publicación, en el mismo año, del Tratado de Medicina y Cirugía Legal de Pedro Mata, varias veces reeditado antes de concluir el siglo ${ }^{1}$.

Este texto, siguiendo la pauta conceptual del alienismo, analizaba algunas desviaciones de la conducta sexual relacionándolas con el cuadro de las monomanías. Como es sabido, esta noción, elaborada originalmente por Esquirol, designaba un tipo de delirio parcial (opuesto al delirio generalizado propio de la manía) que alteraba exclusivamente y en un momento dado, los instintos, las pasiones, la afectividad, dejando intactas las facultades intelectuales, aunque podía llegar a provocar alucinaciones e impresiones ilusorias. No todo comportamiento sexual heterodoxo implicaba la presencia de monomanía. Por eso, en el curso del peritaje legal, el alienista debía determinar si la transgresión sexual era simplemente un delito (violación, estupro, abusos deshonestos, adulterio, corrupción de menores) o se trataba más bien de un signo de locura parcial que excluía toda culpabilidad en el agente. En este último caso se estaría ante una monomanía expresada en los desarreglos de la conducta sexual.

Este tipo específico recibía el nombre de monomanía erótica. Éstas, según Mata, podían ser de dos clases. En primer lugar, aquellas en las que el «impulso generador» es desencadenado por excitaciones conscientes («erotomanía») y, en segundo término, aquellas en las que la iniciativa reside directamente en los órganos genitales ( «satiriasis», «ninfomanía» y «necromanía»)2.

1 El Vade Mecum de Medicina y Cirugía Legal del propio Mata, se había editado en 1844. De 1846 data el concurso público convocado por la Sociedad Económica de Amigos del País de Barcelona para la construcción de un nuevo manicomio. Como se sabe, el concurso lo ganó Pi i Molist, con su proyecto para el asilo de Santa Cruz, que tardaría años en verse realizado

2 Esta clasificación no aparece de repente en el pensamiento de Mata. En la primera edición de su Tratado de Medicina y Cirugía Legal (1844), se alude a la «monomanía», pero todavía no se encuentra una tipología elaborada. Ésta sí se encuentra en la cuarta edición de la obra (1866). Posteriormente, en la edición de 1874, Mata incluirá la «necromanía» o «monomanía cadavérica». Por otro lado, el doctor madrileño discrepaba de Esquirol; éste no consideraba a la «ninfomanía» y a la «satiriasis» como enfermedades mentales, sino como desórdenes puramente físicos (ESQUIROL, Ph. (1838), Des Maladies Men- 


\section{FRANCISCO VÁZQUEZ GARCÍA}

La «erotomanía», cuyo representante literario, rutinariamente mencionado por los alienistas, era Don Quijote, era identificada con una insistente obsesión amorosa por un objeto determinado. En este estado, el sujeto se encontraba completamente sometido al objeto amado, entrando en una incesante intermitencia de afectos, pasando sin interrupción de la alegría al abatimiento. El «erotómano» permanecía siempre en el plano de las representaciones idealizadas; en este aspecto, su conducta no transgredía los límites del pudor. Sin embargo, admitía Mata, no se trataba de un comportamiento inofensivo; podía implicar extravagancias que rompían las convenciones sociales o conducían directamente al suicidio.

En abierto contraste con esta patología, la ninfomanía y la satiriasis no se proyectaban hacia un objeto determinado; su blanco no eran representaciones conscientes idealizadas, sino individuos de carne y hueso. El primer tipo afectaba a los varones; el segundo a las mujeres. El fundamento de estas enfermedades era una exaltación del «aparato genésico». La ninfomanía se consideraba más peligrosa que la satiriasis, pues arruinaba por completo las funciones sociales atribuidas a la mujer ${ }^{3}$. Por otro lado Mata estimaba que la satiriasis, cuya frecuencia se consideraba muy escasa, podía conducir a transgresiones sexuales que rayaban en lo inaudito.

En la última escala de las monomanías eróticas se situaba la «necromanía»o «monomanía cadavérica». Esta patología, ausente en el tratado de Esquirol e inventada a mediados del siglo XIX, lindaba según Mata con otro género de monomanía, la «monomanía homicida o destructora». Designa al irrefrenable impulso de violar sepulturas. Ésta pasión no tenía siempre objetivos sexuales; existían necrómanos, señala Mata, que se limitaban a abrir tumbas para mutilar, dispersar o devorar restos cadavéricos sin ninguna meta sexual ${ }^{4}$.

tales, París, J.B. Baillère, pp. 32-33). Mata, sin embargo, engloba a satiriasis y ninfomanía dentro de lo que designa como «aidomanía», una clase particular de monomanía (Mata, P. (1874), Tratado de Medicina y Cirugía Legal, vol. 2, Madrid, p. 367 y MATA, P. (1868), De la Libertad Moral o Libre Arbitrio, Madrid, pp. 319-321). Otros alienistas españoles tampoco compartían la taxonomía de Esqurol, como es el caso de Giné y Partagás, quien, apoyándose en el concepto de «monomanía impulsiva o instintiva», frente a la noción esquiroliana de «manía impulsiva» (no es un impulso móvil y variable sino una disposición fija y permanente), hace valer la etiología hereditaria de esta enajenación de la voluntad caracterizada «desde la infancia por ciertas irregularidades del carácter, por gustos caprichosos, por instintos perversos» Giné Y PARTAGÁs, J. (1876), Tratado Teórico-Práctico de Frenopatología, Madrid, p. 444. Sobre las peculiaridades nosográficas de Giné, $c f$. DiEGUEZ, A. (1998), «El problema de la Nosografía en la obra psiquiátrica de J. Giné y Partagás», Asclepio, 50 (1), pp. 199-222

3 Me he ocupado de este concepto en VÁZQUEZ GARCÍA, F. (1994) «Ninfomanía y construcción simbólica de la femineidad (España, siglos XVIII-XIX», en CANTERLA, C. (ed.) (1994), La Mujer en los siglos XVIII y XIX. VII Encuentro de la Ilustración al Romanticismo, Cádiz, Universidad de Cádiz, pp. 125-135

4 Sobre la importancia de la «necromanía» en el alienismo español y sobre el debate suscitado en Francia a raíz del caso del sargento Bertrand respectivamente, $c f$. VÁZQUEZ GARCía, F. y MORENO Mengíbar, A. (1997), Sexo y Razón. Una Genealogía de la Moral Sexual en España, Madrid, Akal, pp. 245-47; LANTERI-LAura, G. (1979), Lecture des Perversions. Histoire de leur appropiation médicale, 
Los actos de pederastia o las relaciones carnales con sujetos del mismo sexo quedaban, como tales, fuera del cuadro de las monomanías y por lo tanto del alcance de la medicina mental. Sin embargo Mata, que a la altura de 1870 ya estaba al tanto de las aportaciones procedentes de la nueva psiquiatría degeneracionista ${ }^{5}$, cuyos primeros análisis de la «perversión» comenzaron a conocerse en España durante esa épo$\mathrm{ca}^{6}$, dudaba a la hora de calificar como patológicos ciertos actos de sodomía y de «amor lésbico»: «tal vez deberían figurar aquí como tipos de esas horribles aberraciones ciertos hechos de amor socrático y lésbico, y de sodomía tan fuera del orden común que no parecen posibles en un estado de razón» ${ }^{7}$.

Mata cita como ejemplo histórico el caso del «monstruo infanticida» Gilles de Rais. Estas consideraciones, no obstante, nunca rebasan los límites conceptuales del alienismo clásico y de su tabla de monomanías. Para que la conducta sexual desviada tenga el rango de enfermedad tiene que ser muy excepcional («ciertos hechos (..) tan fuera del orden común») e implicar una pérdida de la razón.

En segundo lugar, la conducta sexual era objeto de examen por parte de una abundante literatura sobre higiene y conservación de la salud. En este caso, el comportamiento sexual desviado solía ser analizado dentro de un capítulo dedicado a la «higiene de la reproducción» ${ }^{8}$. La literatura higienista, desde 1800 hasta las últimas décadas del siglo ${ }^{9}$ trataba este asunto ajustándose a un modelo cuantitativo que per-

Paris, Masson, pp. 17-18 y FouCault, M. (1999), Les Anormaux. Cours au Collège de France 19741975, Paris, Gallimard y Le Seuil, pp. 267-270

5 Sobre la relación de Pedro Mata con el «degeneracionismo», cf. ÁlvAREZ-URíA, F. (1983), Miserables y Locos. Medicina Mental y Orden Social en la España del siglo XIX, Barcelona, Tusquets, p. 185.

6 Así, por ejemplo, el texto de Lassègue sobre el «exhibicionismo» aparece reseñado en $\mathrm{S} / \mathrm{N}$ (1877): «Los exhibicionistas», El Siglo Médico, p. 430. Sobre la noción de «perversión» en el degeneracionismo, $c f$. LANTERI-LAURA (1979), pp. 47-52 y HUERTAS GARCÍA-ALEJO, R. (1990), «El concepto de 'perversiòn' sexual en la medicina positivista», Asclepio, 42 (2), 89-99. CAMPOS, R. (1999), «La Teoría de la degeneración y la profesionalización de la psiquiatría en España (1876-1920), Asclepio, 51 (1), 185203, ha demostrado que la psiquiatría degeneracionista era conocida por los médicos españoles desde la década de 1870, y aplicada en los peritajes médico-legales desde esta época. Sin embargo, su difusión en el ámbito clínico y asistencial sólo comenzó a partir de 1900. FOUCAULT (1999), p. 155-56, por otra parte sitúa entre 1845-1850 el momento en que, en contraste con el limitado papel que le reconocía el alienismo clásico, la «sexualidad» pasó a ocupar un lugar central en el estudio de la enfermedad mental.

7 MATA (1874), vol. 2, p. 377

8 Los términos utilizados varían («higiene de las funciones generadoras», «higiene de la cópula», «higiene de la vida de la especie», «higiene del instinto de propagación», etc..), pero todos ellos connotan que el «instinto sexual» todavía no ha sido conceptualizado como un objeto diferente del «instinto de reproducción». Sólo con la psiquiatría de las perversiones será posible referirse a un «instinto sexual», a una «sexualidad» cuya existencia es independiente de las funciones reproductoras

9 Sin mencionar los textos traducidos (Tourtelle, Hufeland, Deslandes, Descuret, etc.), pueden señalarse los siguientes tratados: HURTADO DE MENDOZA, M. (1839), Instituciones de Medicina y Cirugía, t. I, Madrid, pp. 101-107; RodríGuez GuERrA, A. (1846), El Conservador de la Salud. Manual de higiene pública y privada, Cádiz, pp. 186-199; MoNLAU, P. F. (1865), Higiene del Matrimonio o el Libro 


\section{FRANCISCO VÁZQUEZ GARCÍA}

mitía representar la economía sexual humana. ${ }^{10}$. El uso del «instinto de propagación» implicaba un dispendio de energía que debía mantenerse dentro de ciertos límites si se quería preservar el equilibrio del organismo. El estado patológico consistía en una disminución o aumento en relación con un gasto sexual moderado. Las nociones de «continencia excesiva» y de «incontinencia» (esta última se consideraba más común) engendraban un variado repertorio de enfermedades que iban desde la esfera puramente física (impotencia, esterilidad, tabes dorsal, tuberculosis, ceguera, sífilis, etc..) hasta la mental (pérdida de la razón, histeria, ninfomanía, etc.). El paradigma de este discurso higienista era sin duda la masturbación ${ }^{11}$, verdadera «enfermedad total» ${ }^{12}$ que, en el límite, podía figurar en el cuadro etiológico de cualquier patología. Eventualmente, el «funesto hábito» podía ser inducido en el niño por domésticos o instructores viciosos, pero esta iniciación no se identificaba a su vez con una patología sexual. En este marco conceptual, las conductas sexuales heterodoxas eran acogidas como manifestaciones de excesos cuyo símbolo era el funesto vicio del onanismo, pero no existía ninguna preocupación por catalogar las variedades existentes estableciendo una nosografía a partir de sus diferencias cualitativas.

En tercer lugar, hay que referirse a la práctica de la medicina legal. Obviamente, esta disciplina es, en algunos de sus problemas, una aplicación de la medicina mental, como se ha constatado al hablar de las monomanías. Sin embargo, el dictamen del forense intervenía también en relación con lo que, a mediados del siglo XIX, se designaba como «delitos contra la honestidad», en particular violaciones, estupros, raptos, abusos deshonestos y escándalos públicos que no ponían en liza la posible «demencia» de sus agentes. Los legistas tenían también a su cargo la determinación de la «verdadera identidad sexual» en los casos de duda, es decir, principalmente en los casos de supuesto hermafrodismo.

de los Casados, Madrid, pp. 186-198 y 621-29 (la $1^{\text {a }}$ edición de este difundido texto data de 1853); GINÉ y PARTagÁs, J. (1871): Curso Elemental de Higiene Privada y Pública, Barcelona, pp. 540-551; MoNLAU, P.F. (1875), Elementos de Higiene Privada, t. I, Madrid, pp. 349-355 ( $1^{\mathrm{a}}$ ed. de 1847); ALCINA, B. (1882), Tratado de higiene privada y pública, vol. 1, Cádiz, pp. 511-516 y SANTERO, F.J. (1885), Elementos de Higiene Privada y Pública, Madrid, 1885, pp. 401-411. Sobre higiene y sexualidad en la España del s. XIX, $c f$. CASCO SOLís, J. (1990), «La higiene sexual en el proceso de institucionalización de la sanidad pública española», Asclepio, 42 (2), 223-252

$10 C f$. NYE, R. (1989), «Sex difference and male homosexuality in French medical discourse, 18301930», Bulletin of the History of Medicine, 63 (1), 32-51, p. 34 y NYE, R. (1999), «Introduction» a NYE, R. (ed.) (1999), Sexuality, Oxford, Oxford U.P., p. 12

11 Sobre este asunto existe ya una vastísima bibliografía. Para el caso español, $c f$. PERDIGUERo GIL, E. y GonZÁlez dE PABLO, A. (1990), «Los valores morales de la higiene. El concepto de onanismo como enfermedad según Tissot y su tardía introducción en España», Dynamis, 10, 131-62 y VÁZQUEZ GARCíA, F. y MORENO MENGÍBAR, A. (1997), pp. 94-130

12 FoucAult (1999), p. 223 
En España, el Código Penal de $1822^{13}$, inspirado en los preceptos del napoleónico de 1810 y las sucesivas reformas penales de $1848,1850,1860$ y 1870 , no calificaban como delito el trato carnal entre individuos del mismo sexo, ni reconocían la especificidad del delito de «pederastia». En el código de 1870 aparece el concepto de «abusos deshonestos», diversificado a partir de la vieja categoría de «estupro». En Francia, sin embargo, una ley de 1832 estableció el delito de «paidofilia»; es decir, el placer sexual obtenido con niños, aun sin violencia, pasaba a considerarse delictivo. Esta iniciativa puso en marcha, en el país vecino, una verdadera caza de «paidófilos» o «pederastas»; se consideraba que los abusadores de niños eran siempre individuos con preferencias por los de su propio sexo. El caso más célebre fue el del maestro Ferré, juzgado por paidofilia con sus alumnos en $1843^{14}$. Finalmente, una ley aprobada en 1863 permitía el acto pederástico entre adultos con consentimiento siempre que no mediara escándalo público.

En ese contexto de preocupación por la pederastia se publicó la obra de Ambroise Tardieu, Étude Médico-Légale sur les Attentats aux Moeurs (1857). Este texto contiene un extenso capítulo («De la Pederastia y de la Sodomía») donde por primera vez, de un modo detallado ${ }^{15}$, la medicina afrontaba la tarea de pensar en términos anatómicos (como conformación corporal peculiar) y sociológicos (estilo de vida, costumbres, agrupaciones) lo que más tarde, y ya en términos propiamente psiquiá-

13 El delito de «sodomía», eliminado por el Código Penal de 1822, fue inmediatamente restablecido tras el Trienio Liberal, con la vigencia de la Novísima Recopilación. Habrá que esperar al Código Penal de 1848 para que las relaciones homoeróticas desaparecieran como infracción penal específica. Por esta razón, los tratados de medicina legal anteriores a 1848, como los de PEIRÓ, P. M. y RODRIGO, J. (1841), Elementos de Medicina y Cirugía Legal arreglados a la legislación española, Madrid, p. 77 (1ª ed. 1832) y MAtA, P. (1844), Vade Mecum de Medicina y Cirugía Legal, Madrid, pp. 69-70 recogen la sodomía como un tipo específico de delito; sin embargo, esta noción, como sucedía en en Antiguo Régimen, se refiere a todo «acto contranaturaleza», no exclusivamente a las relaciones con sujetos del mismo sexo. Ejemplo de esto es un caso de sodomía juzgado en Alfaro (Alicante): Gregoria, joven sirviente, declaró en 1846 haber sido penetrada analmente por su señor dos años antes ( S/N (1846), «Dictamen acerca de un presunto atentado de sodomía prestado al juzgado de $1^{\mathrm{a}}$ instancia de la ciudad de Alfaro por sus profesores titulares de medicina, D. Mariano González de Samano y D. Antonio Francés», Boletín de Medicina, Cirugía y Farmacia, 5, 251-52). No obstante, MATA (1844), p. 47, parece reservar el término «sodomía» (o «pederastia», pues los considera sinónimos) para designar el «concúbito de hombre con hombre o de mujer con mujer».

14 Cf. Fouchult (1999), p. 156 y Aron, J.P. y KeMPF, R. (1984), La Bourgeosie, le Sexe et l'Honneur, Bruxelles, Ed. Complexe, pp. 42-44. Otro caso de maestro pederasta fue el de Lessablé, en 1845, cfr. DANET, J. (1977), Discours juridique et perversions sexuelles (XIXe et Xxe siécles), Nantes, Université de Nantes, p. 25. Este autor sitúa la campaña en el contexto de una crítica anticlerical de los internados religiosos.

15 Aunque la conceptualización de la «pederastia» como aberración del instinto sexual aparece ya en la Psychopathia sexualis (1844) de Kaan, en un artículo de Michéa sobre la filopedia (1849) y en otro de Casper sobre violación y pederastia (1852), la descripción más completa de esta conducta y la que tendrá más impacto en la medicina española, será la de Tardieu. 


\section{FRANCISCO VÁZQUEZ GARCÍA}

tricos, se designará como «uranismo» (Ulrich 1864), «homosexualidad» (Benkert 1869), «impulso sexual contrario» (Westphal 1870) e «inversión del sentido genésico» (Charcot y Magnan 1882). En la exploración de este tipo de conducta se sentarán las bases de la futura teoría de las perversiones ${ }^{16}$.

El texto de Tardieu fue traducido por primera vez al castellano en 1863 por los médicos forenses de Madrid, Nemesio López Bustamante y Juan de Querejazu y Hartzensbuch a partir de la tercera edición francesa. Posteriormente, de forma integral o parcial, se volvería a editar en nuestro idioma ${ }^{17}$. Aunque con anterioridad algunos médicos legistas españoles como Pedro Mata habían llegado a ofrecer ciertos detalles sobre el estilo de vida de los pederastas, indicando sus emplazamientos más propicios (cuarteles, cárceles, presidios, buques) y los modos por los que se gestaba este hábito (muchachos corrompidos por la seducción o forzados a prostituirse por la miseria), apenas puede encontrarse en sus tratados un análisis de la fisonomía o un retrato social de estos sujetos. La exposición se concentra casi exclusivamente en la descripción de los signos anatómicos y en la indicación de los gestos médicos que el profesional debe tener en cuenta para detectar, con motivo de denuncias de violación, estupro o abusos deshonestos, la presencia de actos sodomíticos, con un especial interés por las huellas del coito anal.

El tratado de Tardieu, sin embargo, implicaba una alteración profunda de estas maneras tradicionales de la medicina forense. No busca sólo la descripción del acto y de sus vestigios materiales; partiendo de un verdadero archivo etnográfico (más de cien casos observados), traza minuciosamente el perfil social e incluso anatómico del individuo pederasta. Apoyándose en las categorías forjadas por Heinrich Kaan, califica de «perverso moral» al pederasta. Con aval estadístico, destruye ciertos prejuicios (v.g. la idea común de que el matrimonio y el estado de pederastia son mutuamente exclusivos, ciertos tópicos sobre la relación entre hábitos activos y pasivos, etc), pasando del desciframiento de los signos físicos al estudio sociológico de las condiciones de vida, las costumbres, el lenguaje, las formas de asociación y sus códigos. De este modo, los indicios corporales aparentemente más inocentes, los gestos aparentemente más triviales, aparecen como síntomas de un tipo específico y cualitativamente peculiar de conducta sexual. El pederasta se revela sobre todo en su físico, como si sus hábitos denotaran la presencia de una anatomía singular. Se ha pasado de una indagación de huellas que delaten el acto sodomítico a toda una geografía corporal propia de un tipo de sujeto: el pederasta. Así por ejemplo, Tardieu se detiene in extenso en la descripción de las dimensiones y la forma del pene. Los pederastas

16 LANTERI-LAURA (1979) p. 31

17 La referencia a estas diversas versiones de Tardieu al castellano puede encontrarse en VÁZQUEZ García y Moreno Mengíbar (1997), p. 239, n. 105 
poseen con frecuencia «un miembro muy delgado, agudo y afilado por la punta», que «recuerda enteramente el canum more» ${ }^{18}$.

En esta misma época, por otro lado, los médicos comienzan a relacionar la ambigüedad anatómica de los pretendidos hermafroditas con el gusto sexual ambiguo. Algunos sugieren que el segundo no es sino la consecuencia de la primera. Así lo declara Monlau:

\begin{abstract}
«¿Existen en la especie humana verdaderos hermafroditas o individuos que reúnan los dos sexos?. No. Lo que hay es uno que otro varón imperfecto que presenta muchos de los caracteres exteriores de las hembras, así como una que otra hembra con varios de los atributos masculinos. Lo que hay son algunos maricas, u hombres de textura floja, de facciones mujeriles, voz afeminada, carácter tímido y aparato genital poco desarrollado; y también algunas marimachos o mujeres hombrunas (viragines), de costumbres masculinas, voz ronca, barba poblada, clítoris muy abultado»19.
\end{abstract}

Refiriéndose a éstas, señala: «suelen ser de gran talla e inclinadas a procurarse goces ilícitos con las personas de su sexo». En el mismo momento, por lo tanto, tiene lugar un encuentro entre la búsqueda de una anatomía característica de los pederastas y la indagación de un psiquismo peculiar en los supuestos hermafroditas. De aquí nacerán los futuros personajes del «homosexual» y la «lesbiana», conceptualizados como «hermafroditas psíquicos», individuos con cuerpo de varón y alma de mujer o viceversa.

\title{
LA IRRUPCIÓN DEL HOMOSEXUAL
}

Pero a la altura de 1863, cuando se publicó el texto de Tardieu, la medicina forense española no iba aún muy lejos en esta dirección. Se podría pensar que la tardía recepción española de la psicopatología de las perversiones - y por tanto de la noción de «homosexualidad»- que empezaba a constituirse principalmente en Francia y en Alemania a mediados del siglo XIX , tenía que ver con un escaso desarrollo, en nuestro país, de subculturas homoeróticas ${ }^{19 b}$ como las que existían en estas naciones.

18 TARDIEU, A. (1863), Estudio médico-forense de los atentados contra la honestidad, Madrid, p. 140. Las tesis de Tardieu son analizadas en ARON y KEMPF (1984), pp. 47-78 y HuERTAS GARCíAALEJO (1990), 91-94. Se pueden comparar los análisis detallados de Tardieu con la parquedad de MATA, P. (1844), Vade Mecum de Medicina y Cirugía Legal, Madrid, pp. 69-70

19 Monlau (1865) p. 156. Sobre el deslizamiento del estilo anatómico, dominante en el discurso médico sobre el sexo hasta mediados del siglo XIX (y todavía prevaleciente en Tardieu y Monlau), al estilo psiquiátrico, y sobre el papel desempeñado en este cambio por el análisis de los hermafroditas, cfr. DAVIDSON, A.I. (1987), «Sex and the emergence of sexuality», Critical Inquiry, 14, pp. 19-23

19b Sobre el concepto de «subcultura homosexual», cf. GUASCH, O. (1997), «Minoría sexual y sexo disidente. De la práctica sexual a la subcultura», en BUXÁN, X.M. (ed.) (1997), conCiencia de un Singu- 


\section{FRANCISCO VÁZQUEZ GARCÍA}

Por otro lado, la aprobación, en países como Alemania (1870) y Gran Bretaña (1885) de leyes específicamente dirigidas contra la conducta homosexual en privado o en público - ya se constató una dinámica similar en Francia con la aprobación de una ley contra la paidofilia en 1832- y los sonados escándalos a los que esto dio lugar - los casos de Eulenburg en Alemania y de Oscar Wilde en Gran Bretaña- estimuló la investigación en este campo ${ }^{20}$. En cualquier caso la primera razón, referida a la ausencia de subculturas homoeróticas, es difícil de probar mientras no se realicen estudios empíricos sobre el asunto. De momento sólo existen indicios contradictorios. El testimonio de los traductores del texto de Tardieu parece avalar la mencionada tesis de la ausencia: «en España no se ha propagado afortunadamente tan asqueroso vicio del modo en que lo ha hecho en el vecino imperio; y aunque existe, ni ha obligado la intervención de los tribunales por servir de medio preparatorio al homicidio, ni se presenta con tanto cinismo» ${ }^{21}$. Sin embargo Casper, el célebre doctor alemán, se refiere en la misma época y en su tratado de medicina legal, a una «sociedad» de pederastas constituida en Barcelona; por su parte, Teodoro Yáñez, catedrático de Medicina Legal en la Universidad de Madrid, se refiere a un «club de pederastas» que existía en la capital, en una casa de la plaza de Herradores, cuyos miembros eran iniciados a través de elaborados rituales ${ }^{22}$.

Lo que parece claro es que la medicina española acogió con cierto retraso e indiferencia la nueva psicopatología del instinto sexual, derivada tanto de la doctrina degeneracionista francesa como de las nosografías elaboradas en Alemania. Plausiblemente, esta recepción se produjo primero en el ámbito de las aplicaciones médico-

lar Deseo. Estudios lesbianos y gays en el Estado Español, Barcelona, Laertes, pp. 149-166. Este mismo autor ha realizado un importante estudio sobre la subcultura homosexual en España entre el final del Franquismo y la Transición, $c f$. GUASCH, O. (1991), El entendido: condiciones de aparición, desarrollo y disolución de la subcultura homosexual en España, Tesis de Doctorado, Tarragona, Universitat Rovira y Virgili; y GUASCH, O. (1992), La sociedad rosa, Barcelona, Anagrama.

20 HeKMA, G. (1999), «Same-sex relations among men in Europe 1700-1990», en EDER, F., HALL, L. y HEKMA, G. (eds) (1999a), Sexual Cultures in Europe. Themes in Sexuality, Manchester, Manchester U.P., pp. 90-91. No entraremos en la discusión, que recuerda un poco la del «huevo y la gallina», sobre si la «identidad homosexual» fue una pura construcción discursiva de la psiquiatría (tesis de Halperin y Faderman a partir de una lectura demasiado restringida de Foucault) o una realidad social ya diferenciada desde la Baja Edad Media (tesis de Boswell y Trumbach). No obstante, pensamos, con autores como Dollimore y Weeks, que si bien la personalidad «homosexual» es una invención decimonónica —lo contrario sería obviar la condición performativa del lenguaje médico—, su emergencia no es el resultado de cambios puramente discursivos. Los sexólogos y psiquiatras no inventaron desde cero; produjeron unas definiciones que pretendían monopolizar el modelado de cierto tipo de sociabilidad y comportamiento peculiares, colonizar este territorio que se mostraba con intensidad en relación directa con el crecimiento urbano. Sobre este debate, $c f$. WEEKS, J. (1985), El Malestar de la Sexualidad. Significados, mitos y sexualidades modernas, Madrid, Talasa Ed., pp. 155-159

21 TARDIEU (1863), p. 125, n. 1

22 Citado en ARON y KEMPF (1984), pp. 76-77 y YÁÑEZ, T. (1884), Elementos de Medicina Legal y Toxicología, Madrid, p. 323, respectivamente 
legales de la psiquiatría y sólo más tardíamente — a partir de comienzos de sigloen la esfera propiamente clínica.

La nueva psiquiatría, en la que se encuadraba la teoría de las perversiones sexuales, surgió en Europa en torno a 1850-1870, rompiendo con las antiguas categorías del alienismo. En esta tradición, la clave de la enfermedad mental era el delirio, aunque fuera «parcial», como en el caso de las «monomanías»; el loco se caracterizaba por estar sumido en el error, de ahí la irresponsabilidad de sus actos. En la nueva psiquiatría, desde la época de Griesinger, Baillarger, Kaan, Morel, y posteriormente Magnan y Lombroso, la clave del estado de anomalía se situaba en el instinto. Era la cuestión de lo voluntario sometido a lo involuntario del instinto, de la falta de autocontrol, del predominio de las funciones cerebrales inferiores, más primitivas y animales, sobre las superiores, más evolucionadas; perturbaciones entendidas ahora como detenciones, retrasos o regresiones en el desarrollo evolutivo individual. La preocupación fundamental de esta nueva psiquiatría no era tanto la curación de enfermos mentales como la detección de anormales; es decir, de sujetos que han transgredido alguna norma social y a los que, sin que exista pérdida de lucidez mental, se puede atribuir una subordinación de la voluntad a las tendencias instintivas. El problema de la responsabilidad es reemplazado por la interrogación acerca del grado de peligrosidad ${ }^{23}$.

Uno de los dominios preferentes del nuevo discurso psiquiátrico era el de la psicopatología sexual. Ésta se ocupaba de la identificación, clasificación y explicación de las distintas perturbaciones del instinto sexual. La conducta sexual se identificaba con la manifestación de un instinto. En primer lugar, y esto implicaba una novedad respecto a la noción clásica de «instinto de propagación», lo propio del instinto sexual era su independencia respecto a las funciones reproductoras. Por eso se entendía que el primero se encontraba ya en los niños, antes de que éstas hicieran su aparición. En segundo lugar, el instinto sexual buscaba satisfacerse, y esta satisfacción entrañaba un placer. Ahora bien, el intermediario entre el instinto y el placer producido era la imaginación. El impulso sexual se satisfacía mediante la asociación de imágenes. En tercer lugar, el instinto sexual se inscribía en un desarrollo, una evolución. Ésta obedecía a una ley, a una determinada teleología. La evolución normal consistía en la progresiva subordinación del instinto sexual al instinto de reproduc-

23 Cf. Foucault (1999), pp. 291-92. Foucault señala que el «delirio» no desaparece en la nueva psicopatología, sino que más bien es reformulado; las variantes del delirio se explican a partir de ahora por la «economía del instinto y del placer» (id., p. 294). Por otro lado, es obvio que la transición del concepto de «monomanía» al de «perversión», tal como lo elaboran los degeneracionistas, no se produce de golpe. LANTERI-LAURA (1979), p. 58 y FouCAUlT, M. (1981), «L'evolution de la notion d'individu dangereux dans la psychiatroe légale», Déviance et Societé, 5, pp. 403-422, p. 414, han emplazado el concepto de «locura moral» (desarrollado por los psiquiatras británicos Prichard y Ray) en un lugar intermedio. Sobre la evolución desde el concepto de «monomanía» hasta el de «conciencia mórbida» pasando por el de «locura moral», $c f$. Álvarez PElÁEZ, R. y HuERTAS, R. (1987), ¿Criminales o locos?, Madrid, CSIC, pp. 43-48 


\section{FRANCISCO VÁZQUEZ GARCÍA}

ción. Esto exigía someter las tendencias originariamente indiferenciadas e involuntarias del impulso sexual a una diferenciación paulatina y a un creciente control de la voluntad. La anormalidad sexual consistía por una parte en un desequilibrio por el que las funciones cerebrales superiores, asociadas a lo voluntario, se subordinaban a las funciones inferiores, asociadas al imperio de lo involuntario. Por otra parte, toda anormalidad era vista como una regresión o detención evolutiva; el retroceso a una fase más primitiva de desarrollo, con el predominio de las funciones más simples o animales, o el estancamiento en una etapa determinada ${ }^{24}$.

La elaboración teórica de cada una de estas consideraciones abría nuevas posibilidades. Permitía por una parte unificar la captación médica de las conductas sexuales, superando las disparidades entre las conceptualizaciones de la medicina mental, la higiene y la medicina legal: todas las desviaciones sexuales, desde la masturbación hasta la violación homicida, pasaban a engrosar el campo de las «anomalías» del instinto sexual. Éstas, además, podían ser catalogadas como especies cualitativamente distintas, rebasando el modelo puramente cuantitativo del viejo higienismo ${ }^{25}$. Por último, la doble inscripción de estas anomalías en la relación de lo voluntario con lo involuntario y en el desarrollo filogenético y ontogenético (por lo tanto en la herencia), permitía explicarlas a la vez desde un modelo neurológico y desde las leyes de la biología evolutiva ${ }^{26}$. El organicismo y el evolucionismo de la nueva psicopatología sexual tienen aquí su raíz.

Antes de 1900, este tipo de discurso sólo se encontraba en la psiquiatría española de un modo fragmentario y, como se ha dicho, más frecuentemente en la esfera médico-legal que en el ámbito estrictamente clínico. Las categorías que el viejo alienismo había forjado para comprender las desviaciones sexuales, en particular la noción de «monomanía erótica»o «genésica» resistieron aquí durante mucho tiempo ${ }^{27}$. No es raro encontrarla mezclada con el nuevo concepto de «perversión sexual», incluso en los tratados y peritajes de medicina legal publicados en España durante las dos últimas décadas del siglo XIX.

24 Id., pp. 293-96.

25 Pensamos con LANTERI-LAURA (1979), pp. 26-29 y FOUCAULT (1999), p. 282 y en desacuerdo con NyE (1989), p. 40 y NYE, R. (1993): Masculinity and Male Codes of Honor in Modern France, New York, Oxford, Oxford U.P., pp. 101-102 que la tipología de las perversiones implica el tránsito de un modelo cuantitativo a un modelo cualitativo en la representación de la sexualidad.

26 FOUCAULT (1999), p. 289.

27 ÁlVAREZ-URÍA (1983), pp. 184-88. No hay que olvidar que el célebre peritaje de Esquerdo en el caso de Garayo el Sacamantecas, diagnosticaba en el mismo la presencia de una «monomanía genésica», aunque a la vez indagaba los antecedentes hereditarios y estigmas físicos del sujeto, en afinidad con las prácticas del degeneracionismo. $C f$. ESQUERDO Y ZARAGOZA, J. ( 1881), «Locos que no lo parecen. Garayo 'el Sacamantecas', en REY GONZÁLEZ, A.M. (1990), Estudios Médico-sociales sobre marginados en la España del siglo XIX, Madrid, Ministerio de Sanidad y Consumo, pp. 224, y CAMPOS MARÍN (1999), p. 191. 
Un buen ejemplo de esta situación lo proporciona el Tratado de Antropología Médica y Jurídica en dos volúmenes (1889-1894) del Dr. Ignacio Valentí y Vivó (1841-1924), catedrático de Medicina Legal y Toxicología de la Universidad de Barcelona, fundador de la Academia de Higiene de Cataluña y uno de los pioneros del movimiento eugénico en Cataluña. Si se compara su aproximación a las desviaciones sexuales con la de otros tratados de la época, por ejemplo los Elementos de Medicina Legal y Toxicología (1884) de Teodoro Yáñez, catedrático de la misma disciplina en la Universidad de Madrid, se advierten diferencias sustanciales. Yáñez sigue aferrado al cuadro de las «monomanías eróticas», y, en relación con la pederastia, se apoya completamente en Tardieu ${ }^{28}$. Valentí y Vivó, en cambio, incorpora ya toda una nueva familia de conceptos y de referencias:

\footnotetext{
«Para esclarecer los signos de la venere prepostera, saphica, felatrix en el acusado y la víctima, son de utilidad los datos consignados en las obras de Tardieu; pero comparándolos con otros posteriormente obtenidos por Hofmann, Maschka, Weiss, Tamassia, Lassègue, Fournié, Charcot, Westphal, etc., al procurar el diagnóstico de los estados que se denominan: exhibicionismo, inversión del instinto sexual, feminismo [sic], tribadismo y otras aberraciones de parecida calaña, propias de la enfemedad neurofrénica y del vicio en la más ínfima ralea de gentes, que viven brutalmente asociadas y en libertad inmerecida ${ }^{29}$.
}

Este autor hace valer las nociones de la nueva psiquiatría («degeneración», «herencia mórbida», «anomalía órgano-dinámica», «enfermedad cerebro-medular», «aginesia», «disgenesia») asociando incluso la proliferación de «aberraciones sexuales» con el declive de la civilización («amenazada de retroceso por este solo peligro de la brutalidad carnal») $)^{30}$. Al mismo tiempo, viejos conceptos como el de «erotomanía» perviven sin acabar de reformularse (se señala que esta noción ha sido mal definida al asociarla con la ninfomanía y la satiriasis, pero no se ofrece una elaboración alternativa como la expuesta por Binet en 1887) ${ }^{31}$.

El panorama cambia ostensiblemente desde comienzos de siglo. La plena acogida de la nueva psicopatología de las perversiones, y en particular de los análisis del homosexualismo, se ubica por otra parte en un contexto singular. La literatura del regeneracionismo surgida a raíz de los últimos desastres coloniales españoles, utiliza

\footnotetext{
28 YÁÑEZ (1884), pp. 173-179. Sobre Valentí y Vivó, cf. ÁlVAREZ-PElÁEZ, R. (1988), «Origen y desarrollo de la eugenesia en España» en SÁNCHEZ Ron, J. M. (1988), Ciencia y Sociedad en España, Madrid, CSIC y El Arquero, p. 184.

29 VAlentí y ViVó, I. (1889), Tratado de Antropología Médica y Jurídica, t. I, Barcelona, p. 414

$30 I d .$, p. 408.

31 Id., p. 409. Sobre la reformulación de la «erotomanía» por Binet, NYE (1989), p. 41. Esta misma mezcolanza de restos conceptuales procedentes del alienismo con nuevas categorías derivadas de la psicopatología de las perversiones, se manifiesta en las novelas naturalistas española publicadas en esa misma época, $c f$. FERNÁNDEZ, P. (1997), «Scientia sexualis y saber psiquiátrico en la novela naturalista decomonónica», Asclepio, 49 (1), pp. 240-41.
} 


\section{FRANCISCO VÁZQUEZ GARCÍA}

con frecuencia una retórica que se vale de las metáforas del «debilitamiento físico»o de la «desvirilización» y del «afeminamiento» para diagnosticar los males de la patria. Éstos se ven como una consecuencia de la pérdida de voluntad, de la abulia colectiva. El derrumbe del país es vivido en parte como una crisis de la masculinidad ${ }^{32}$. En segundo lugar, existen algunos testimonios sobre la existencia de subculturas homoeróticas consolidadas en las principales ciudades de España ${ }^{33}$. En tercer lugar

32 Las referencias serían innumerables. Joaquín Costa, por ejemplo, consideraba que «España era una nación unisexual, compuesta de dieciocho millones de mujeres»; más tarde recitificó: «es una nación sin sexo. No es una nación de mujeres, es una nación de eunucos» (cit. en TIERNo GALVÁN, E. (1971), «Costa y el Regeneracionismo» en Escritos (1950-1960), Madrid, Tecnos, p. 462. Refiriéndose a Madrid como un «falansterio de degenerados», Macías Picavea exclama: «iQué ideas! ¡Qué pasiones! ¡Qué feminismo! Los que vamos de provincias, aún más toscos y zafios, echamos en seguida de ver la falta de salud mental, de virilidad afectiva, de equilibrio. ¡Y semejante desconcierto es lo que nos gobierna!» (Picavea, M. (1996), El Problema Nacional, Madrid, Biblioteca Nueva, p. 302). Mallada recuerda que «a ser cierto que el pueblo español posee menor virilidad en el presente que en otros tiempos pasados» (MalladA, L. (1994): Los Males de la Patria y la Futura Revolución Española, Madrid, Alianza Editorial, p. 37). Altamira, por su parte, recuerda que la tarea de la Universidad ha de ser «crear generaciones de ánimo viril, que no se apoquen ante las dificultades comunes a todos los pueblos» (ALTAMIRA, R. (1997), Psicología del Pueblo Español, Madrid, Biblioteca Nueva, p. 191); Ramiro de Maeztu clamaba contra la reinante «moral de tullidos» y se valía del siguiente símil para indicar el talante de los intelectuales que necesitaba la nación: «las mujeres prefieren los hombres bien nutridos a los golfos escuálidos y a los poetas decadentes» (De MAEZTU, R. (1997), Hacia otra España, Madrid, Biblioteca Nueva, p. 222). Entre los síntomas de la «degeneración» española, Isern parece aludir explícitamente a la homosexualidad: «degeneradas aquellas ciudades en las cuales reviven por modo especial los vicios de la decadencia de Grecia y Roma y, en especial, el estetismo» (Del Desastre Nacional y sus Causas, cit. en Tierno Galván (1971), p. 418). No hay que olvidar que, un año antes de la publicación de este libro, en plena marea del Desastre de Cuba, se suscitó un escándalo, que trascendió a la prensa nacional. Se reveló que, de forma soterrada, el servicio de Higiene Especial del Gobierno Civil de Cádiz toleraba y regulaba la prostitución masculina homosexual. En la prensa se habló entonces de los «afeminados», de los «estetas» como «vergüenza de la viril raza española». El asunto terminó con la dimisión del Gobernador Civil, cuñado del mismísimo Antonio Maura ( $c f$. Moreno Mengíbar, A. y VÁzQuez García, F. (1999), Crónica de una Marginación. Historia de la prostitución en Andalucía desde el siglo XV hasta la actualidad, Cádiz, Biblioteca Andaluza de Arte y Literatura, pp. 77-78. Los regeneracionistas relacionaban esta falta de energía viril con la «flojedad de cuerpo» característica de la «raza española». Mallada se refiere a este rasgo (MALLADA, L. (1998), La Futura Revolución Española y Otros escritos regeneracionistas, Madrid, Biblioteca Nueva, pp. 294-97) y resalta su presencia en la aristocracia, degenerada por los «goces del sensualismo» (if., p. 310) y en la burguesía, que, en comparación con la de otras naciones «tiene menos virilidad, porque se alimenta peor y vive al día más desarregladamente, sin el espíritu de ahorro» (id., p. 323). Por esta razón, autores como Iradier de Herredo (Hacia un nuevo tipo de español, Madrid, 1917) o Luis Morote consideran que «en la educación física estará el remedio» de esta carencia de salud y vigor. Sobre la «abulia», cf. GANIVET, A. (1957), Idearium Español, Madrid, Austral, pp. 131-38.

33 Aunque queda por realizan un análisis en profundidad, se cuenta ya con algunos indicios que testimonian la existencia de estas «sociedades secretas» en algunas ciudades españolas desde el reinado de Alfonso XII. Ya se han mencionado las informaciones de Casper y Yáñez sobre Madrid y Barcelona, respectivamente (vid. supra, n. 22). Rodríguez Solís, utilizando la prensa madrileña de 1890 , se refiere a una banda de muchachos «que tenían por jefe a un caballero bien vestido», dedicados a «llevar engaña- 
hay que referirse a la preocupación - muy extendida entre los reformistas sociales de principios de siglo- por la «infancia en peligro», y en particular por la corrupción sexual de los niños, dentro de una estrategia más general que pretendía convertir a la familia en un instrumento para el gobierno de las poblaciones ${ }^{34}$. No hay que olvidar que se atribuía a los homosexuales una irresistible inclinación paidófila. Por último, la imaginería anticlerical de comienzos de siglo tendía a asociar cada vez más al sacerdote con la pederastia y con la iniciación homófila ${ }^{35}$. En estas condiciones no es de extrañar que se acrecentara el temor y el interés por el personaje del «homosexual». Sin embargo, salvo en diagnósticos médico-legales como el aludido en el anexo 2 de este trabajo, el término predominante - en la literatura especializada y más aún en los medios de comunicación- a la hora de designar esta perversión no era

dos por la noche al Prado a hombres incautos», a los que posteriormente chantajeaban; «por entonces descubriéronse varias casas, verdaderas Sodomas y Gomorras de nuestros días, en las que se encontraron niños de cierta edad llevados allí con engaños por hombres malvados» (RoDRíGUEZ SoLís, E. (s.a.), Historia de la Prostitución en España y en América, Madrid, p. 295; la edición que utlizamos parece datar de 1921). En fecha algo posterior, el abogado Joaquín del Moral menciona, también en Madrid, la existencia de «falsos perseguidores» de sodomitas (¿se trata de policías corruptos?), conocidos con el nombre de «ronda del ful» que en realidad negocian con «tan asqueroso vicio» (DEL MORAL Y PÉREZ ALOE, J. (1913), El Estado y la Prostitución, Madrid, pp. 84-85). Otro testimonio referido a la España de Alfonso XII: «cuenta Sales Mayo en su libro La Condesita, la existencia de una secreta sociedad conocida como 'Sociedad San Guiñolé', en la que se daban cita los elegantes y sofisticados de la época que tuvieran aficiones sáficas o sodomíticas. Eran de la alta clase y tenían sus particulares signos diferenciales, pertenecían al Círculo Mercantil, al Casino o a la Ópera (..) En la Calle del Calvario y en la del Horno de la Mata fueron encontrados muchos de estos 'guiñolistas' elegantes, entre chulos y canallas de los barrios bajos. El más famoso de los lugares de encuentro de la época estaba en la calle de la Alameda. Cuenta Rodríguez Solís que en aquél lugar había un baile conocido por 'El Ramillete', y en una redada de una noche de Carnaval durante el noctámbulo reinado de Alfonso XII se detuvieron a más de cien de aquellos chicos» (RIOYO, J. (1991), Madrid. Casas de Lenocinio, Holganza y Malvivir, Madrid, Espasa Calpe, pp. 281-82). Por nuestra parte, hemos investigado la existencia de prostitución masculina homosexual en Cádiz y Sevilla entre 1880 y 1920 en MoRenO MENGíbar y VÁzQueZ García (1999), pp. 176-178 y VÁZQuEZ García, F. y MoRENO MENGÍBAR, A. (1998), Poder y Prostitución en Sevilla, t. II, Sevilla, Pub. Universidad de Sevilla, pp. 219-20.

34 Sobre la preocupación por la infancia en la España de comienzos de siglo, existe ya una amplia bibliografía. Entre las publicaciones más recientes, se pueden citar algunos de los trabajos contenidos en BORRÁS LlOP, J.M (dir.) (1996), Historia de la Infancia en la España Contemporánea 1843-1936, Madrid, Ministerio de Trabajo y Asuntos Sociales y Fundación Germás Sánchez Ruipérez y el artículo de HuERTAS, R. (1998), «Niños degenerados. Medicina mental y regeneracionismo en la España del cambio de siglo», Dynamis, 18, 157-179. Sobre la «corrupción sexual» de la infancia, me remito a la investigación que para su tesis doctoral lleva a cabo bajo mi dirección el profesor José Benito Seoane: La Pasión y la Norma. Una genealogía de la moral sexual infantil en la España del siglo XIX, Universidad de Cádiz.

35 CONARD, P. (1971), «Sexualité et Anticlericalisme (Madrid, 1910)», Hispania. Revista Española de Historia, 117, 103-34. Este mismo componente anticlerical del discurso homófobo (y de las campañas contra la masturbación de adolescentes y el histerismo femenino) se daba en Francia (DANET (1977), p. 25 y NYE (1989), pp. 38-39). El caso mencionado en el anexo 2 corresponde precisamente a un sacerdote. La novela naturalista española de finales del XIX se ubica en esta misma dirección, asociando al clero con la vesania sexual (FERNÁNDEZ, P. (1997), «Moral y scientia sexualis en el siglo XIX. El eros negro de la novela naturalista», Analecta Malacitana, 11, pp. 192-94). 


\section{FRANCISCO VÁZQUEZ GARCÍA}

el de «homosexual», sino el de «invertido», como por otra parte sucedió en el resto de Europa, al menos hasta $1914^{36}$. La preferencia por esta expresión podía ser de origen alemán («Die conträre Sexualempfindung» de Westphal 1870, o el libro con el mismo título, publicado por Möll en 1891) o francés (la «inversion du sens genital» de Charcot y Magnan 1882), aunque la forma sustantivada (el «invertido sexual») aparece por primera vez en el británico Havelock Ellis (1896), cuyos Studies in the Psychology of sex se vertieron parcialmente al castellano entre 1906 y $1913^{37}$.

Entre 1900 y 1920, coincidiendo con la incorporación de las teorías degeneracionistas y lombrosianas y el despegue de la «cuestión sexual» ${ }^{38}$, se produjo en España la difusión a amplia escala de la nueva psicopatología de las perversiones ${ }^{38 b}$. Se es-

36 NYE (1989), p. 43 n. 50. En la documentación administrativa y hemerográfica que hemos utilizado para estudiar la prostitución en Cádiz y en Sevilla entre los siglos XIX y XX, los términos más usuales son los de «afeminado» (v.g. «hombres de aspecto y costumbres afeminados»), «esteta» (cuyo origen no está claro, pues se encuentra en la prensa de 1898, de manera que es improbable que su filiación se remonte a la «inversión sexo-estética» descrita por Ellis hacia 1896) y, algo más tarde (hacia 1910) «inversión», que por otra parte parece ser el término más utilizado por los psiquiatras españoles desde 1900. No obstante, unas Instrucciones impresas y difundidas en 1909 por la Jefatura Técnica de la Higiene Especial en Cádiz, referida a los burdeles, señalaban que «tampoco se consentirá la permanencia en la casa de chulos o individuos que viven a costa de las mujeres, ni de individuos de aficiones homosexuales» (cit. en MORENO MENGíBAR y VÁZQUEZ GARCÍA (1999), p.179)

37 El posible que el término se introdujera a través de la obra de Albert Möll, traducida al castellano con el título de Las Perversiones del Instinto Sexual, Madrid, 1896. Por otra parte, la primera versión castellana de la Psicopathia Sexualis de Krafft-Ebing se realizó a partir de la versión francesa, en la edición comentada y ampliada por Albert Möll: KRAFFT-EBING, R. (1955): Psicopatía Sexual, Buenos Aires. Sexual Inversion, primera parte de los Studies in the Psychology of Sex de Havelock Ellis, se publicó en inglés en 1897 y en alemán en 1896, pero la edición inglesa fue retirada por las autoridades judiciales (WEEKS, J. (1978), «Havelock Ellis y la Política de la Reforma Sexual» en Rowbotham, S. Y WEEKS, J. (1978), Dos pioneros de la liberación sexual. Edward Carpenter y Havelock Ellis. Homosexualidad, feminismo y socialismo, Barcelona, Anagrama, pp. 168-69

38 Cleminson, R. M. y AMEZÚA, E. (1999), «Spain: the political and social context of sex reform in the late nineteenth and early twentieth centuries» en Eder, F. X., Hall, L. \& Hekma, G. (1999b), Sexual Cultures in Europe. National Histories, Manchester, Manchester U.P., pp. 173-96

$38 \mathrm{~b} \mathrm{El}$ análisis en profundidad de estas teorías, queda fuera de los márgenes del presente trabajo. Pueden ofrecerse, no obstante, algunas indicaciones. A comienzos de siglo, Simarro recurre indistintamente a los conceptos de «locura moral» $\mathrm{y}$ «degeneración» para explicar las «perversiones e inversiones sexuales» (SIMARRO, L. (1900), «Sobre el concepto de la locura moral», Revista Iberoamericana de Ciencias Médicas, 3, pp. 396-99, p. 398. La degeneración será invocada deste esta época para explicar, tanto la delincuencia precoz como la homosexualidad. Cf. SANCHÍs BANús, J. (1916): Estudio médico-social del niño golfo, Madrid; RODRÍGUEZ LAFORA, G. (1917), Los niños mentalmente anormales, Madrid y SALAS VACA, J. (1920), Los degenerados en sociedad, Madrid. Los autores más próximos a Lombroso, por su parte, invocan la «detención de desarrollo», bajo las formas del «infantilismo» o el «atavismo» como causas de la criminalidad sexual o «de significación generativa» (SALILLAS, R., «Delimitation de la nature pathologique du délit» en Comptes Rendues du XIV Congrès International de Médécine, Madrid, Avril 23-30, 1903, Madrid, pp. 660-671. Bernaldo de Quirós no duda en señalar el valor preventivo de los escritos realizados por el «invertido sexual» para conocer con antelación su conducta criminal (BERNALDO de QUIRÓS, C. (1900), «Delincuentes que escriben», Revista Iberoamericana de Ciencias Médicas, 4, 
cribieron algunas monografías médico-legales sobre las relaciones entre criminalidad y perversión. Los «invertidos» aparecían presentados como principales responsables de delitos de escándalo y de los abusos deshonestos y violaciones sufridos por los niños. Por otro lado, cada vez se hizo más frecuente la presencia de homosexuales en los relatos literarios ${ }^{39}$. La crónica de sucesos y la sensibilidad pública, acrecentada y alarmada ante lo que se contemplaba como una oleada de prostitución masculina, hacían el resto:

«En España, el cura Meliá, y los atentados contra el pudor de niños, el del Chato del Escorial en el niño Pedrín, son casos bien patentes de estos crímenes impuros. Sin llegar a crímenes, el Juzgado de guardia casi diariamente entiende en lesiones graves, y los jueces municipales podrían dar una estadística, mejor que nosotros, de los escándalos que originan lo mismo los auténticos sodomitas que sus falsos perseguidores, llamados la ronda del ful. Estos hacen un comercio de tan asqueroso vicio, convirtiéndose en proxenetas de hombres para hombres» ${ }^{40}$.

Pero los «invertidos», y con ellos toda la familia de los perversos, no eran solamente un asunto de la psiquiatría en su vertiente forense. La propia literatura estrictamente clínica se ocupaba del asunto. Un buen ejemplo lo proporciona la Guía del Diagnóstico de las Enfermedades Mentales (1900) de Martínez Valverde, una de las obras más representativas del degeneracionismo español. En este texto, de modo sistemático, se adoptan los postulados de Magnan a la hora de conceptualizar las perversiones. Estas enfermedades son afrontadas como «transtornos de los instintos» o «estigmas de degeneración»e implicaban una «desharmonía de las funciones cerebrales»:

«Estas depravaciones genésicas revisten la forma de sodomía, bestialidad, safismo, necrofilia o violación de cadáveres, mutilaciones voluntarias, ninfomanía, onanismo (este último en especial en los idiotas en imbéciles), y otras muy diversas obsesiones eróticas, de las cuales una de las más notables por su importancia social y que, por tanto, merece alguna más detenida atención, es la inversión del sentido sexual (..) ; generalmente recae tal aberración en sujetos que tienen un estigma hereditario, esto es, son unos verdaderos degenerados» ${ }^{41}$.

pp. 509-512, p. 510. Sobre la difusión de la antropología lombrosiana en España, $c f$. GALERA, A. (1991), Ciencia y Delincuencia. El determinismo antropológico en la España del siglo XIX, Sevilla. Sobre la polémica entre lombrosianos y degeneracionistas, $c f$. NYE, R. (1984), Crime, Madness and Politics in Modern France. The medical concept of National Decline, Princeton, Princeton U.P., pp. 98-131.

39 Monografías médico-legales: VALENTí Y VIVÓ, J. (1911), Criminales lujuriosos y Agresividad psicosexual, Barcelona, y PIGA PASCUAL, A. (1912), Relaciones entre la Lujuria y las Perturbaciones del Instinto Sexual, Toledo. Este último, en las pp. 28-31, cita a Krafft-Ebing y relaciona la «inversión sexual» con el abuso de menores. Sobre la presencia literaria, $c f$. CRUZ CASADO, A. (1995), «La homosexualidad en algunas narraciones españolas de principios de siglo (1900-1930)», El Bosque, 10-11, pp. 187-199. Sin embargo, aunque la muestra estudiada se emplaza en un periodo algo posterior, Jean-Louis Guereña ha señalado la casi total ausencia de temática homosexual masculina en la literatura erótica española, en contraste con la abundante presencia de la homosexualidad femenina (GUEREÑA, J.L. (1999), «De Erotica Hispanica», Cahiers d'Histoire Culturelle, 5, p. 31).

40 Del Moral y PÉrez Aloe (1913), pp. 84-85.

41 Martínez Valverde, J. (1900), Guía del Diagnóstico de las Enfermedades Mentales, Barcelona, pp. 33-34. Sobre la inscripción «degeneracionista» de este texto, cf. CAMPOS MARín (1999), pp. 200- 


\section{FRANCISCO VÁZQUEZ GARCÍA}

Como es sabido, la difusión de las tesis degeneracionistas permitía ofrecer una explicación biologista del declive nacional. Pero, más específicamente, ¿qué implicaciones políticas poseía este enfoque psicopatológico y fuertemente organicista de la homosexualidad? Como es sabido, en Alemania e Inglaterra, que contaban con leyes explícitamente homófobas, el discurso médico sobre la homosexualidad, funcionó de manera ambivalente; en unos casos legitimaba nuevas formas de persecución y control; en otros casos permitía justificar la homosexualidad como un hecho de la naturaleza, un tendencia innata que no podía por ello ser legalmente castigada. Las primeras tentativas de organización política emprendidas por la minoría homosexual respondieron a la voluntad de derogar la legislación persecutoria y para ello se apoyaron en el discurso psiquiátrico ${ }^{42}$.

En España, sin embargo, las cosas parecen haber sido diferentes, aunque nuestro análisis se detiene en los primeros años del siglo XX. Es posible que, al no existir leyes antigays - la primera correspondió al Código Penal de 1928, que no llegó a aplicarse- tampoco existieran iniciativas políticas al estilo de la alemana o la británica para organizar a la minoría concernida. Tampoco parece que existieran voces aisladas que - como sucedió en Francia con Zola y Gide ${ }^{43}$ — defendieran la causa homosexual. Es probable que el discurso psiquiátrico sobre la homosexualidad se limitara principalmente a desempeñar funciones estigmatizadoras, aunque este extremo debería ser comprobado en un estudio posterior ${ }^{43 \mathrm{~b}}$ Esta herencia recibida habría abortado, en buena medida, la posibilidad de que se gestara en España un movimiento político de resistencia homosexual; no parece haber existido nada semejante en el periodo anterior a la Guerra Civil, ni siquiera en los sectores más avanzados del anarquismo español, muy innovadores en el campo de la política sexual ${ }^{44}$. El futuro político de este colectivo parecía sellado.

201. El libro de GALCERÁn i GRANÉS, I. (1908), Diagnóstico Frenopático, Gerona, también se asienta en la teoría de la degeneración, aunque el desarrollo del asunto es mucho más limitado (se trata de un folleto) que en el texto de Martínez Valverde.

42 HeKma (1999), pp. 84-88 y MCLAREN, A. (1999), «National responses to sexual perversions: the case of transvestism», en Eder, Hall y Hekma (1999a), pp. 132-34.

43 NYE (1989), pp. 46-48. Una posible excepción puede ser el caso del escritor Álvaro Retana, quien a comienzos de los años 30 descalificó las concepciones homófobas de Marañón y Jiménez de Asúa. $C f$. Cleminson, R. (1999), «Male homosexuality in Contemporary Spain: signposts for a sociological analysis», Paragraph. A Journal of Modern Critical Theory, 22 (1), pp. 35-36.

43b Un ejemplo de este actitud en el discurso psiquiátrico puede encontrarse en los artículos sobre la homosexualidad publicados en la revista Sexualidad dirigida por el Dr. Antonio Navarro Fernández. Dejo constancia de mi agradecimiento al profesor Richard Cleminson por permitirme consultar su trabajo inédito titulado: «Sexualidad (1925-28), psychiatry and homosexual panic».

44 Cleminson, R. (1995), Anarquismo y Homosexualidad, Murcia, Huerga y Fierro eds., pp. 150-51. 


\begin{abstract}
Anexo no 1
«Oficio ${ }^{45}$ del Teniente de Alcalde del $2^{\circ}$ Cuartel a la Alcaldía:

En la tarde del cuatro del corriente, avisado por José Ruiz, vecino del número 10, calle del Maizal, el alguacil de esta Tenencia José Coldión para que diese auxilio a fin de detener a un hombre que con empeño se había llevado a un niño pequeño de Ruiz a una casa de lenocinio de la calle del Carmen, conocida por la de «la Valenciana», y solicitado hacer obscenidades con el referido párvulo. En seguida se dirigió el alguacil a la casa y condujo detenido al hombre, que es Manuel Montemayor, vecino del $n^{\circ} 83$ calle de la Soledad, al que fue necesario trasladarlo a la Prevención de la Guardia Civil por los gritos que daba y desvergüenzas que profería (..). La opinión pública marca como demente al expresado Montemayor. Dios que a V.S. guarde, Cádiz, 6 de julio 1846. Juan Rafael Durán
\end{abstract}

Carta $^{46}$ del padre de Montemayor al Alcalde:

Montemayor, natural y vecino de esta ciudad, a V.S. con el respeto debido expone: que como aparece de la solicitud que en 28 de abril último hizo a esa Alcaldía (..) solicitó y le fue concedido recoger a su hijo Manuel en el refugio de Capuchinos con la condición de que si el estado de su demencia se acrecentase, fuese trasladado a la Casa Hospicio donde estuviese recogido y asistido como tal demente por no permitir otra cosa sus desórdenes y manías que ya tocan en crímenes. La ocurrencia de su fuga de otro asilo por dos ocasiones; el escándalo dado la noche del 21 del pasado en que quería (..) asesinar al exponente; los desórdenes y estafas que está cometiendo por todo el vecindario; el acontecimiento del Sábado último (..) acreditan la imperiosa necesidad de que V.S. se sirva disponer que se lleve a efecto el entero de la referida solicitud (..) Cádiz, 6 de julio, 1846. Antonio Montemayor.

[al margen]: reconozcan los facultativos titulares a Montemayor y justifiquen si está o no demente. Fdo. José López».

Parte Facultativo ${ }^{47}$ :

En cumplimiento de la orden de V.S. hemos reconocido en la Prevención Civil a D. Manuel Montemayor con objeto de averiguar si está o no demente: por las contestaciones que se nos han dado a las preguntas y cuestiones que le hemos hecho, no parece que Montemayor padezca semejante enfermedad; el encargado del local donde aquél se encuentra detenido manifiesta que en los días que Montemayor está en [ilegible] no ha observado acción ni otro que acredite padecimiento de locura. Sin embargo no por esto podrá decidirse que el citado Manuel no padezca esta enfermedad, si como asegura su padre es intermitente; en este concepto V.S. podrá disponer si Montemayor debe estar en observación o acreditar que está efectivamente loco para que pueda como tal ser trasladado al Departamento de los supuestos asignados (..) Cádiz, 9 de julio, 1846»

45 Archivo Municipal de Cádiz, C/1.131 (R). Se trata de un cuadernillo ( $n^{\circ}$ 71, 1846) titulado «Personas Escandalosas que no caben en el Mundo» que contiene oficios y correspondencia varia. Este oficio es el documento $\mathrm{n}^{\circ}$ 5. En este anexo sólo incluimos una selección.

$46 I d$., documento $\mathrm{n}^{\circ} 6$.

47 Id., documento $\mathrm{n}^{\circ} 7$. 


\section{FRANCISCO VÁZQUEZ GARCÍA}

\section{$\operatorname{Anexo} \mathbf{n}^{0} 2^{48}$}

«La circunstancia de no haber encontrado en las obras recientes contemporáneas y ya clásicas de Westphal, Laségue, Charcot, Magnan y von Krafft-Ebing, por no citar más que a los grandes maestros, un caso análogo al que voy a exponer ante esta Sección es lo que me impele a molestar la atención de los sabios que la forman, por si con su análisis se aumenta el acervo de la Medicina Legal, si he de exponer las nociones, los síntomas que tengan un carácter científico innegable en el examen de este pervertido sexual, de los cuales, en general, aún la medicina no ha mucho se apartaba con horror faltando al noble deber de descubrirlos y defenderlos, por abominables, inmorales e inmundos que parezcan los actos por ellos cometidos a los ojos del mundo ignorante» (..).

Trátase de un sujeto (U.L.), de 41 años de edad, sacerdote, de los Bajos Pirineos (Francia), que sorprendido en uno de los frecuentes tratos sexuales que tenía con niños, fue denunciado a la Administración de Justicia, incoándose el correspondiente sumario y viéndose la causa ante el Tribunal del Jurado, que apreció: 'se trataba de un degenerado que padece un estado de locura parcial limitada, en orden a sus aficiones sexuales, que le impulsa contra su voluntad a realizar actos de la índole de los que eran objeto de la acusación y le priva en absoluto de la libertad de apartarse de ejecutarlos'; y no obstante, la Sala, estimando precisa para la irresponsabilidad del procesado la existencia de un estado total completo de locura común a todos los actos de la vida, sólo otorgó a la afirmación del Jurado el valor de una circunstancia atenuante, condenando al procesado en su consecuencia (..).

Resumiendo todo lo que llevamos dicho, podemos afirmar que los antecedentes fisiopatológicos de la familia y herencia de este individuo, pueden sintetizarse de este modo: cortos de vista (miopes), el padre, la madre, el hermano mayor y el degenerado que examinamos; enfermedades nerviosas: el padre y una tía paterna, la madre y la abuela paterna; estigmas psíquicos: sus hermanos, Pablo, grandezas; Adriano, avaro y taciturno; José, conocido por la 'Mariposa', y sus dos hermanas. También encontramos enfermedades constitucionales, infecciosas e intoxicaciones en los ascendientes (..) integrándole en lo físico algunos estigmas, entre los cuales descuella la asimetría de su cerebro, que es platicéfalo (..) y su constitución eminentemente escrofulosa (..) en síntesis, este sujeto presenta desórdenes en los sentimientos y en la voluntad, con obsesiones e impulsiones y estigmas físicos muy ostensibles, y debe ser considerado como un degenerado mental, congénito, abúlico, con manifestaciones psicopáticas homo-sexuales, caracterizadas por dedicarse a la masturbación, de preferencia con la boca, sufriendo la erección y experimentando la voluptuosidad de la eyaculación cuando realiza tan inmorales maniobras e inmundas succiones».

48 El texto que sigue es una selección del trabajo presentado por BRAVO Y MORENO, F. (1904), «Exposición de un caso clínico médico-legal de psicopatía homo-sexual» en Comptes Rendues du XIV Congrés Internationale de Mèdecine, publiés sous la direction de M. le Dr. A. Fernández-Caro, Secretaire Général du Congrés. Section de Médecine Légale et Toxicologie, Madrid, pp. 96-102 